\title{
Invariant Manifold of Symmetric Orbits for Globally Optimal Biped Locomotion
}

\author{
Sang-Ho Hyon ${ }^{* * *}$ Kenji Fujimoto ${ }^{* * *}$ \\ * JST, ICORP, Computational Brain Project, Kyoto, Japan \\ ** ATR, Computational Neuroscience Laboratories, Kyoto, Japan \\ (e-mail:sangho@atr.jp) \\ *** Graduate School of Engineering, Nagoya University, Nagoya, Japan \\ (e-mail: fujimoto@nagoya-u.jp)
}

\begin{abstract}
This paper proposes novel walking gait generation for a double-pendulum-like biped walking model based on the family of the symmetric orbits. By introducing an involution $\mathrm{R}$ associated with the leg switching map, the flow of the saddle-center dynamics of the uncontrolled pendulum possesses time-reversal symmetry with respect to $\mathrm{R}$, and the conjunction of the flow and $\mathrm{R}$ forms a family of symmetric orbits parameterized by the orbital energy and the stride. The invariant manifold of the family of symmetric orbits is obtained numerically or approximately using a perturbation method. The proposed methods are evaluated on numerical simulations. By constraining the solution onto the invariant manifold using the control inputs, stable walking gaits are generated in a semi-global manner in simulations. Based on the passivity of the closedloop system, a robust speed-controlled walking is achieved in a very simple way.
\end{abstract}

Keywords: Biped locomotion, Hamiltonian systems, Time-reversal symmetry, Symmetric orbits, Invariant manifold, Passivity, Numerical simulation

\section{INTRODUCTION}

The goal of this paper is to generate robust and energyefficient locomotion gaits that achieve simple targets such as a specified walking speed or desired locations. For that purpose, we propose a novel motion control strategy based on the useful properties of Hamiltonian systems. As outlined in Hyon (2005), the strategy comprises three steps:

S1. First disregard dissipativity of the system to extract purely Hamiltonian (conservative) dynamics. Then, apply an invariance controller that renders the closedloop system lossless and restricts the hybrid solutions into invariant sets.

S2. Retrieve (and inject as necessary) dissipativity and apply tracking controllers to stabilize asymptotically target states or periodic orbits passing through them.

S3. Apply adaptive or learning schemes to minimize control efforts.

Roughly speaking, S1 is introduced to make biped robot behave as a ball rolling on the ground, by way of feedback control. The resultant controller has a hierarchical structure in which the existence, stability, and boundedness of the solution are ensured by the lowest layer with a high priority; more advanced control goals are achieved at higher layers.

In this paper, we treat a double-pendulum biped model and propose the semi-global ${ }^{1}$ locomotion generation.

\footnotetext{
1 The "semi-global" means we consider stability at once. Since configuration space is limited in biped locomotion (foot cannot penetrate the ground), the stability is naturally limited to semiglobal.
}

Specifically, we utilize the symmetric orbit inherent in the uncontrolled system. The symmetric orbits (see Sevryuk (1986) or Lamb (1998)) are special orbits in (time-) reversal systems such as Hamiltonian systems. They exist as a family parameterized by the initial conditions such as the Energy level (imagine flows around the center of a simple pendulum dynamics), and form an invariant manifold in the phase space.

Symmetric orbits are not necessarily periodic. However, if we allow switching between phase variables, they, together with the switching map, can form some meaningful hybrid periodic orbits on an invariant manifold. Consequently, by constraining the solution curves to the invariant manifold using control inputs, (semi-) global orbit generation would be possible within the admissible region. In this case, external forces just change the internal energy of the system, which results in robust gait generation. This is the motivating idea of this study.

The idea of constraining the solution to some parameterized family of orbits is not itself new in locomotion literatures. For example, Wieber (2006) proposed to use whole walking trajectories computed off-line, and to select one of them online. Although not mentioned about symmetric orbits, Morita (2004) proposed a ballistic walking based on the symmetry. Hyon (2005) also studied symmetric orbits parameterized by the step length and angular momentum, by using numerical method based on Poincaré map. In Hyon (2005), however, the authors had no idea how to represent and store the obtained infinitely many symmetric orbits. Therefore, the authors picked up a simplest target manifold $\left(q_{1}+q_{2}=0\right)$ and constrained the solution to the manifold by control inputs. Although the controller 
was almost same as the past-proposed one Grizzle (2001) we believe Hyon (2005) is the first paper that addressed its (semi-) global stability of the symmetric orbits on the manifold, from a global dynamics point of view. The idea was also validated on a real biped walking robot. Once obtained the global stability, it was easy to obtain global asymptotically stable periodic gaits, by using a very simple feedback controller ${ }^{2}$.

This paper extends our previous work toward globally optimal walking gait generation by the following two novel approaches. First, we compute approximate, but analytical invariant manifold in which symmetric orbits are filled in. We do this because, once the invariant manifold has been obtained analytically, it is easy to apply model-based controllers to constrain the solution onto the manifold. Actually, in this paper, we employ two kinds of continuous feedback controllers. One is a simple trajectory tracking controller, and the other is a velocity-field tracking controller in Duindam (2004), resulting in the second contribution of this paper. The effectiveness of the proposed methods are evaluated on numerical simulations.

This paper is organized as follows. In Section 2 we introduce the reversal symmetry and symmetric orbits of Hamiltonian systems and explain the close link to the periodic orbits which we are interested in. In Section 3, we clarify the relationship between biped walking and symmetric orbits, by introducing a double-pendulum (compass-like) biped model. We obtain the symmetric orbits numerically, and explain their properties. In Section 4 we approximately compute the invariant manifold of the symmetric orbits. In Section 5, we propose a sub-optimal gait generation based on the symmetric orbits and its invariant manifold. In particular, we show the application of the method by Duindam (2004), which maintains the energy constant, while tracking to some given tangent vector field. In Section 6, we examine how the proposed method is applicable to realistic walking model with dissipation through simulations, then demonstrate that a very simple passivity-based speed controller can make the robot walk stably at arbitrary speed. Section 7 summarizes the paper.

\section{REVERSAL SYMMETRY AND SYMMETRIC ORBITS IN HAMILTONIAN DYNAMICAL SYSTEMS}

Hamiltonian systems have a special symmetry, called (time-) reversal symmetry Devaney (1976)Lamb (1998). Let $F: \mathcal{M} \rightarrow \mathcal{M}$ be a diffeomorphism on a manifold $\mathcal{M}$, and a map (called involution) $R: \mathcal{M} \rightarrow \mathcal{M}$ such that $R \circ R=$ id (identity). If

$$
R \circ F=F^{-1} \circ R
$$

is satisfied, we say $F$ has reversal symmetry with respect to $R$ Lamb (1998) (or simply say $F$ is $R$-symmetric).

Let us consider a Hamiltonian system with the Hamiltonian

$$
H_{0}(q, p)=\frac{1}{2} p^{T} M(q)^{-1} p+U_{0}(q) .
$$

2 Experiments and simulation movies are available from http://www.cns.atr.jp/ sangho/
Its flow is represented by $\phi_{t}:\left(q\left(t_{0}\right), p\left(t_{0}\right)\right) \mapsto\left(q\left(t_{0}+t\right), p\left(t_{0}+t\right)\right)$. Then, $\phi_{t}$ is $R$-symmetric since $H_{0}$ is invariant with respect to a map $R:(q, p) \mapsto(q,-p)$. That is, we have

$$
R \circ \phi_{t}=\phi_{-t} \circ R \text {. }
$$

The fixed point sets, $\operatorname{Fix}(R)$ and $\operatorname{Fix}\left(R \circ \phi_{t}\right)$, are especially important because a family of orbits, called symmetric orbits, pass through these sets and are mapped onto themselves by $R$ with their direction of flow reversed. Therefore, if we have a $R$-symmetric flow $\phi_{t}$, and if its fixed point sets $\operatorname{Fix}(R)$ and $\operatorname{Fix}\left(R \circ \phi_{t}\right)$ are identified, we obtain the associated symmetric orbits. The symmetric orbits exist as a family, which is parameterized by initial condition such as initial energy.

The symmetric orbits are not necessarily periodic orbits. However, if they are closed orbits, they pass $\operatorname{Fix}(R)$ exactly twice Devaney (1976). Such symmetric orbit we call symmetric periodic orbit. The Reversible Lyapnov Center Theorem is related to the existence of the symmetric periodic orbits. See Devaney (1976) or Sevryuk (1986).

However, note that a compass-like walking system shown in Section 3.1 has no double center equilibrium because the center equilibrium is out of the admissible configuration space. Instead, it has a saddle-center (a pair of saddle and center) as the meaningful equilibrium. Therefore, a double-pendulum has no meaningful symmetric periodic orbits in nature. However, if we allow us to have a hybrid dynamical system framework, where the continuous states and discontinuous states coexist, hybrid symmetric orbits can be newly defined, and they play a central role in global generation of periodic orbits. As we will see in Section 3.2, this scenario is actually possible if $R$ represents a leginterchanging map.

\section{SYMMETRIC ORBITS OF BIPED WALKING MODEL}

\subsection{Model description}

Let us consider a walking model shown in Fig. 1. This is often called "compass model", and is a double pendulum as long as its foot sticks to the ground all the way, which we assume in this paper. We take the angles of the swinging leg and supporting leg as the generalized coordinates, $q=\left(q_{1}, q_{2}\right)$, on the admissible configuration space $\mathcal{N}:=\left\{q \in S^{2}|| q_{1} \mid \leq \pi / 2\right\}$. The conjugate momentum is $p=M(q) \dot{q} \in \mathcal{M}=T^{*} \mathcal{N}$, where

$$
\begin{aligned}
& M(q)= \\
& {\left[\begin{array}{cc}
\left(m_{1}+2 m_{2}\right) L^{2}+m_{2}(L-b)^{2} & -m_{2} b L \cos \left(q_{1}-q_{2}\right) \\
-m_{2} b L \cos \left(q_{1}-q_{2}\right) & m_{2} b^{2}
\end{array}\right]}
\end{aligned}
$$

is the inertia matrix. The potential energy is given by

$$
U_{0}(q)=\left(m_{1}+2 m_{2}\right) g L \cos q_{1}-m_{2} g b\left(\cos q_{1}+\cos q_{2}\right)
$$

with $g$ being the gravity acceleration. Then, the dynamics of the double pendulum is 2-DOF Hamiltonian system

$$
\begin{aligned}
& \dot{q}={\frac{\partial H_{0}(q, p)^{T}}{\partial p}}^{\dot{p}}=-{\frac{\partial H_{0}(q, p)^{T}}{\partial q}+B\left(u-D{\frac{\partial H_{0}(q, p)^{T}}{\partial p}}^{T}\right)}^{T}+
\end{aligned}
$$




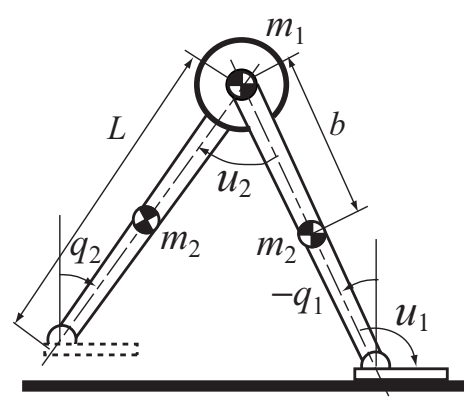

Fig. 1. A simple biped robot with the mass-less foot: $q_{1}$ and $q_{2}$ are the absolute angle of the supporting leg and swinging leg respectively.

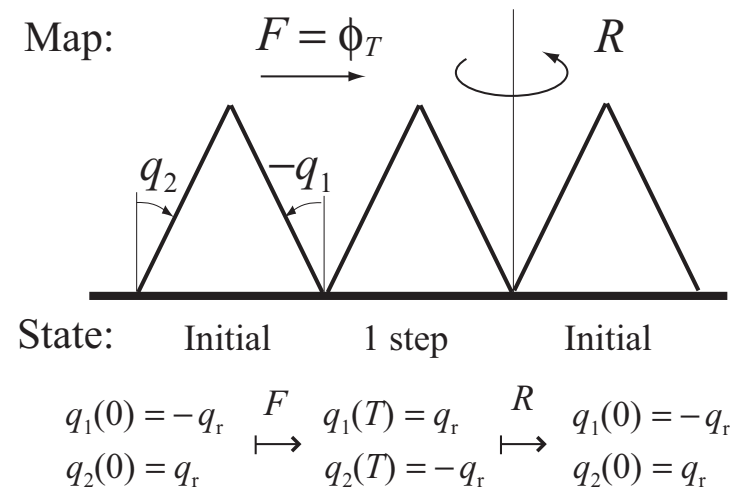

Fig. 2. Gait transition of symmetric walking. The map $F$ is defined in (6). A family of steady symmetric walking gaits is the fixed point set of $R \circ F$.

with the Hamiltonian (2), where $D=$ is a friction coefficient matrix (diagonal), $u=\left[u_{1}, u_{2}\right]^{T} \in \mathbb{R}^{2}$ is the control input vector, and $B=\left[\begin{array}{cc}1 & -1 \\ 0 & 1\end{array}\right]$ is a driving matrix which converts $u$ to the generalized input associated with $q$. The equilibrium (origin) is a saddle-center, and the eigenvalues are $\{\lambda,-\lambda, i \omega,-i \omega\}$ with real numbers $\lambda, \omega$.

\subsection{Numerical search of symmetric orbits}

The model in the previous section represents the dynamics of the single support phase. When the swinging leg lands on the ground, the energy is lost and sudden change of the velocity occurs. Energy will be lost due to the joint friction too. Along with the strategy (S1) in Section 1, however, we ignore the dissipation tentatively (we get back to this point in Section 6), and try to derive a gait generating controller by focusing on the pure Hamiltonian systems.

For this purpose, let us consider a $R$-symmetric flow $\phi_{t}$ of the free system; (4) with $D=0, u=0$. Herein, we are interested in the mirror map about $p$-axis:

$$
R:\left(q_{1}, q_{2}, p_{1}, p_{2}\right) \mapsto\left(-q_{1},-q_{2}, p_{1}, p_{2}\right),
$$

rather than the conventional involution $R:(q, p) \mapsto$ $(q,-p)$. As can be seen in Fig. 2, for some symmetric walking gaits which start $q_{1}(0)=-q_{2}(0)=-q_{r}$ at $t=0 \quad\left(q_{r}\right.$ is some positive number representing the stride) and terminate at $q_{1}(T)=-q_{2}(T)=q_{r}$ at time $t=T$, the involution (5) corresponds to the ideal leg- interchanging $\operatorname{map}^{3},\left(q_{1}, q_{2}, p_{1}, p_{2}\right) \mapsto\left(q_{2}, q_{1}, p_{1}, p_{2}\right)$, in which the impulsive effect is completely ignored. Such orbits are $R$-symmetric orbits which pass the origin of the configuration space (included in the $\operatorname{Fix}(R)$ ). Therefore, it is easy to see we can obtain (ideal) symmetric walking gaits by conjunction of $R$ and $R$-symmetric flow $\phi_{t}$. For this construction making sense, we need to consider flows

$$
F:=\left.\phi_{t}\right|_{t=T}
$$

not arbitrary ones. Wherein, $T>0$ is the time which one cycle of walking finishes (at the end $q_{1}+q_{2}=0$ is met). Therefore, the problem of finding symmetric walking gaits is to find the fixed point set $\operatorname{Fix}(R \circ F)$.

The top graph of Fig. 3(a) shows the numerically found symmetric orbits (Hyon (2004)) associated with the fixed points of Poincaré maps (Guckenheimer (1983)) with discrete jump (Kousaka (1999)). The robot parameters are $\left\{m_{1}=2, m_{2}=1.2, b=0.2, L=0.4\right\}$. The stride is fixed to $q_{r}=0.5$ here, but similar results can be obtained for other values. Fig. 3(b) shows the bifurcation diagram of the eigenvalues of the linearized Poincaré map DP at $\operatorname{Fix}(R \circ F)$, where the bifurcation parameter is the momentum $p_{1}$. As intuitively expected, the (hybrid) symmetric periodic orbits for uncontrolled system without dissipation are found to be neutrally stable or unstable (see Hyon $(2005)){ }^{4}$

Some notes are in order:

- The orbits approach a straight line $q_{1}+q_{2}=0$ as the energy increases and $\dot{q}_{2}$ becomes negatively large. In this case, the walking speed is high, but, in reality, is limited by unilateral constraint and friction cone, which we ignore in this paper for simplicity.

- If the energy is high and $\dot{q}_{2}$ is positively large, the orbit is curved to the $q_{2}$-direction. A particular orbit passes the singular point $q_{1}=0, q_{2}=\pi$. Interestingly, this "giant swing" orbit is free from the impulse at touchdown (lies in the null space of the impulse map). Of course, this is not a walking gait.

- In regions $\left|q_{1}\right| \geq \pi / 2$ and $\left|q_{2}\right|<\left|q_{1}\right|$, walking is indefinable.

Although the region is limited, the symmetric walking gaits fill a certain area of the configuration space. In the 4-dimensional phase space, they form a 3-dimensional invariant manifold.

The global gait generation developed in this paper is achived by constraining all the solutions to this invariant manifold using control inputs. Once the solutions are constrained on the manifold, the control inputs also converge to zero (in ideal lossless system).

\section{APPROXIMATION OF INVARIANT MANIFOLD OF SYMMETRIC ORBITS}

Let us approximate the invariant manifold. We assume the family of symmetric orbits can be approximated by

\footnotetext{
3 We need this map when we define the coordinate as the angles for supporting leg and swinging leg, not those for right leg and left leg 4 Asymptotical stability is impossible in Hamiltonian system in nature. See Guckenheimer (1983).
} 

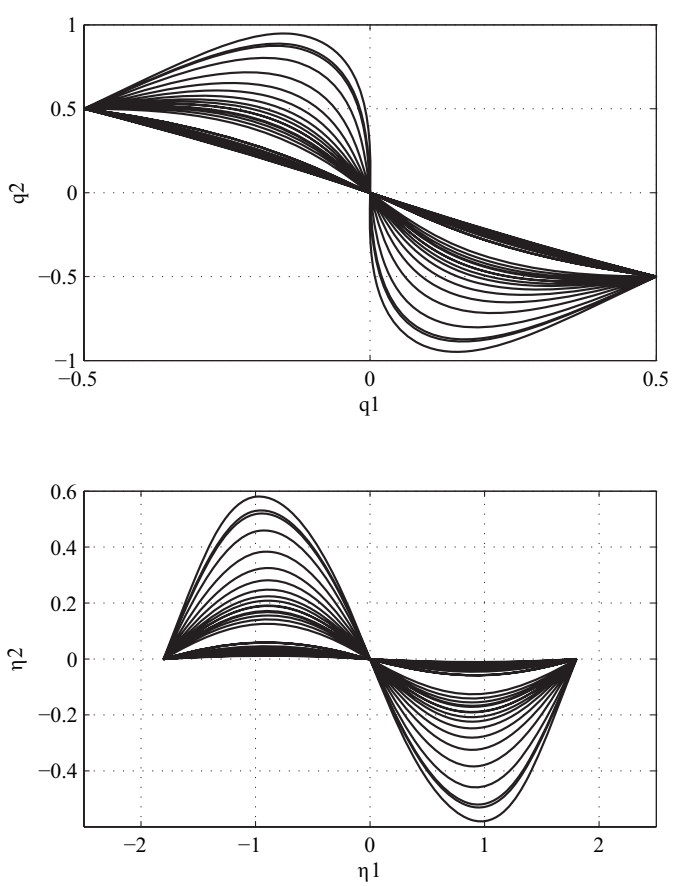

(a) A family of symmetric orbits in the original configuration space (top) and the transformed configuration space (bottom)

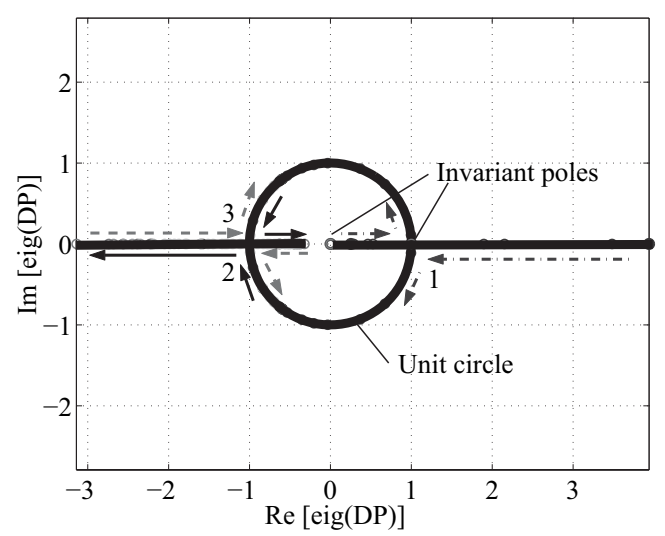

(b) Bifurcation of characteristic multipliers of $\operatorname{Fix}(R \circ F)$ for (5) and (6)

Fig. 3. Numerical computation of the family of symmetric orbits for the simple biped model with the parameters: $\left\{m_{1}=2, m_{2}=1.2, b=0.2, L=0.4\right\}$ and $\left\{q_{1}^{*}=-0.5\right.$, $\left.-10 \leq p_{1}^{*} \leq 0\right\}$

$$
\eta_{2}=A \sin \left(\omega \eta_{1}\right)
$$

via a transformation $Y:\left(q_{1}, q_{2}\right) \rightarrow\left(\eta_{1}, \eta_{2}\right)$, where $\omega=\frac{\pi}{\eta_{r}}$ and $\eta_{r}=(1+\gamma) q_{r}$. This shows curves passing three points $\left(\eta_{1}, \eta_{2}\right)=(0,0),\left(0, \pm \eta_{r}\right)$. This assumption is obtained from the numerical results in the previous section. We apply perturbation method up to terms of necessary order. As the transformation $Y$ we use

$$
\left[\begin{array}{l}
\eta_{1} \\
\eta_{2}
\end{array}\right]=\left[\begin{array}{cc}
\gamma & -1 \\
1 & 1
\end{array}\right]\left[\begin{array}{l}
q_{1} \\
q_{2}
\end{array}\right]
$$

By setting a limit $A>0$, the symmetric orbits meet $\left|q_{2}\right|<$ $\left|q_{1}\right|$ in region $\left|q_{1}\right|<q_{r}$. The orbits Fig. 3(a) is transformed to Fig. $3(\mathrm{~b})$ in the new coordinate $\left(\eta_{1}, \eta_{2}\right)$. It should be noted that the controlled symmetric orbit in Hyon (2005) is a single orbit $\eta_{2}=q_{1}+q_{2}=0$, which corresponds to "scuffing gait". Contrary, (7) meets $\eta_{2}=q_{1}+q_{2}=0$ only at the origin or the leg interchanging configuration $q_{1}=-q_{2}= \pm q_{r}$, as long as $A \neq 0$. The uncontrolled system does not have any solutions with $A=0$ (i.e. $\eta_{2}=q_{1}+q_{2}=0$ is met uniformly). The amplitude $A$ codes the energy level, and is uniquely determined if $\dot{q}_{1}(0)$ or $\dot{q}_{2}(0)$ are given together with the stride and initial energy.

Now, we compute the velocity field $v(\eta)=\left[v_{1}\left(\eta_{1}\right), v_{2}\left(\eta_{1}\right)\right]^{T}$ tangent to the invariant manifold. From reversal symmetry this meets $v\left(-\eta_{1}\right)=v\left(\eta_{1}\right)$. We take a similar procedure for computing center manifold (Guckenheimer (1983)) up to terms of necessary order. The invariant manifold is represented by a power series parameterized by the velocity $v_{10}=\left.v_{1}\right|_{\eta_{1}=0}$ (alternatively, the energy and direction) at which the curves pass $q_{1}=-q_{2}=q_{0}$ and $q_{1}=-q_{2}=q_{r}$.

We first expand (7) to yield

$$
\eta_{2}=b_{1} \eta_{1}+b_{3} \eta_{1}^{3}+\ldots
$$

Then, its tangent vector is represented by

$$
\begin{aligned}
& v_{1}=c_{0}+c_{2} \eta_{1}^{2}+c_{4} \eta_{1}^{4}+\ldots \\
& v_{2}=d_{0}+d_{2} \eta_{1}^{2}+d_{4} \eta_{1}^{4}+\ldots
\end{aligned}
$$

where $c_{0}=v_{10}$. By differentiation, we have

$$
\begin{gathered}
\dot{v}_{1}=\left(2 c_{2} \eta_{1}+4 c_{4} e t a_{1}^{3}+\ldots\right)\left(c_{0}+c_{2} \eta_{1}^{2}+\ldots\right) \\
\dot{v}_{2}=\left(2 d_{2} \eta_{1}+4 d_{4} e t a_{1}^{3}+\ldots\right)\left(d_{0}+d_{2} \eta_{1}^{2}+\ldots\right)
\end{gathered}
$$

Then, these equations are substituted into the dynamics, then solved for the coefficients $\left\{b_{i}, c_{i}, d_{i}\right\}$ using the terminal conditions $\eta_{2}\left(\eta_{r}\right)=\eta_{2}\left(-\eta_{r}\right)=0$. From this procedure we approximately obtain the vector field $v(\eta)=$ $\left[v_{1}\left(\eta_{1}\right), v_{2}\left(\eta_{1}\right)\right]^{T}$. We used Maple for the symbolic computation.

\section{CONSTRAINT CONTROL TO THE INVARIANT MANIFOLD}

We generate semi-globally stable walking gaits by constraining the integral curves to the approximated invariant manifold (4). If the approximation is precise enough, it is expected that the resultant closed-loop solutions maintain the energy level, and that the control inputs become close to zero. The resultant walking gaits would be optimal with respect to the criteria

$$
J=\frac{1}{2} \int_{0}^{T}\|u\|^{2} d t
$$

for given stride. However, the gaits would not be optimal if we consider the energy dissipation due to the touchdown.

Here we will show simulation results by using two kinds of controllers. The one is a simple trajectory tracking 

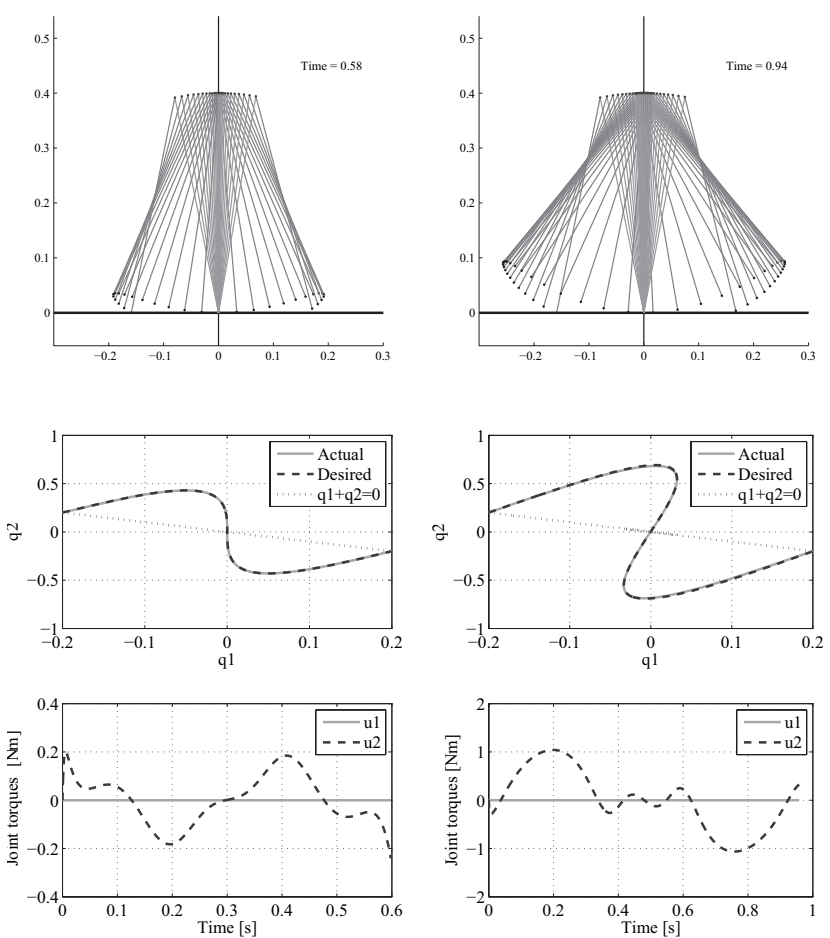

Fig. 4. Controlled optimal and non-optimal symmetric walking gaits. (Left): Starting from a numericallyobtained fixed point. (Right): With the same initial conditions except for $\dot{q}_{2}(0)$, which is manually perturbed. Note that the control input in the left figure is small, but not zero due to the approximation error of the invariant manifold.

controller in the under-actuated case (Section 5.1), where we don't use the tangent vectors but use only the trajectories in configuration space. The velocity is uniquely determined from the available energy of the system. The other controller utilizes the tangent velocity field, instead of using the trajectory itself (Section 5.2). If the initial conditions are the same, and the precision is good, the both results will be identical.

\subsection{Trajectory tracking for under-actuated case}

Let suppose the ankle torque is unavailable, $u_{1}=0$, but only the hip torque $u_{2}$ is available. The controller is simple as follows. First, we select a symmetric orbit represented by $(7)$. Then, we compute the tangent vector by

$$
v_{2}\left(\eta_{1}\right)=-A \omega \cos \left(\omega \eta_{1}\right) \dot{\eta}_{1}
$$

Then, we transform (7) and (15) to the target trajectory $q_{d}, \dot{q}_{d}$ by the inverse of (8). Finally, we apply a feedback controller

$$
u_{2}=-K_{1}\left(q_{2}-q_{2 d}\right)-K_{2}\left(\dot{q}_{2}-\dot{q}_{2 d}\right),
$$

where $K_{1}, K_{2}$ are tracking gains. In this setting, the approximation error will appear in $u_{2}$.

Fig. 4 is the simulation result with $q_{r}=0.2$, where two different gaits are shown. The left graph shows the result with the initial conditions $\dot{q}_{1}(0)=1.7086, \dot{q}_{2}(0)=3.4944$, which are picked up from the numerical solution. The right graph shows the result with $\dot{q}_{2}(0)=5$ and $A$ computed from

$$
A=-\frac{1}{\omega} \frac{v_{10}+v_{20}}{\gamma v_{10}-v_{20}} .
$$

In both case, the orbits are $R$-symmetric, and have symmetry about the walking direction. They always pass the origin and do not fall. But only the former one lies on the approximated invariant manifold, not the latter one (it is not uncontrolled symmetric orbit). The energetic performance is clearly different. We can see, however, that a bit control effort is still required even for the former case, due to the approximation error.

\subsection{Full-actuated maneuver on tangent vector field of invariant manifold}

If the robot is not under-actuated, we have additional DOF for any other control purpose than the trajectory tracking. Here we adopt a velocity field tracking control proposed by Duindam (2004). By using this method, we can maintain the kinetic energy constant while making the solution follow the desired velocity field. We use this because it satisfies our purpose of utilizing an important property of Hamiltonian systems - energy preservation. If we give the well-approximated tangent vector of the invariant manifold as the target velocity field, the closedloop solutions of this controller maintain the energy level, and the control inputs converge to zero. In other words, the solution evolves along the geodesic flow (see Marsden (1999)) of the original uncontrolled system, without falling.

Let $v(q) \in T \mathcal{N}$ be the target velocity field. The controller comprises the following two steps. The first controller is given by

$$
u_{n}=M(q) \frac{\langle\dot{q}, v\rangle^{2}}{\langle v, v\rangle^{2}} \nabla_{v} v-M(q) \frac{\langle\dot{q}, v\rangle\left\langle\nabla_{v} v, \dot{q}\right\rangle}{\langle v, v\rangle^{2}} \nabla_{v} v
$$

Wherein, $\langle v, w\rangle=v^{T} M(q) w$ is the inner product on Riemannian manifold. $\nabla_{v} w$ is the covariant derivative (see Marsden (1999)) of $w$ along with $v$, which can be represented in local coordinate

$$
\left(\nabla_{v} w\right)^{i}=v^{j} \frac{\partial w^{i}}{\partial q^{j}}+\Gamma_{j k}^{i} v^{j} w^{k}
$$

with the connection coefficient $\Gamma_{j k}^{i}$. Note that $\Gamma_{j k}^{i}=$ $\frac{1}{2} m^{i l}\left(\frac{\partial m^{k l}}{\partial q^{j}}+\frac{\partial m^{l j}}{\partial q^{k}}-\frac{\partial m^{j k}}{\partial q^{l}}\right)$ is the Christoffel symbol of the second kind $\left(m^{i j}\right.$ is the element of the inverse of the inertia matrix). The controller (18) ensure $\dot{q}(t)=v(q(t))$ as long as the initial velocity $\dot{q}_{0}$ is in parallel to $v\left(q_{0}\right)$, and the cross product of the input and the velocity becomes to zero: $\left\langle u_{n} \mid \dot{q}\right\rangle=0$, that is, energy is preserved.

A second controller is given by

$$
u_{a}=\beta M(q) \frac{\langle\nu, \nu\rangle \operatorname{sign}\langle\dot{q}, v\rangle}{|v|} v-\beta M(q) \frac{|\langle\dot{q}, v\rangle|}{|v|} \nu
$$

with appropriate tracking gain $\beta$. Wherein, $\nu$ is a vector such that ${ }^{5}$

\footnotetext{
5 we used $\alpha=\frac{\langle\dot{q}, v\rangle}{\langle v, v\rangle}$.
} 

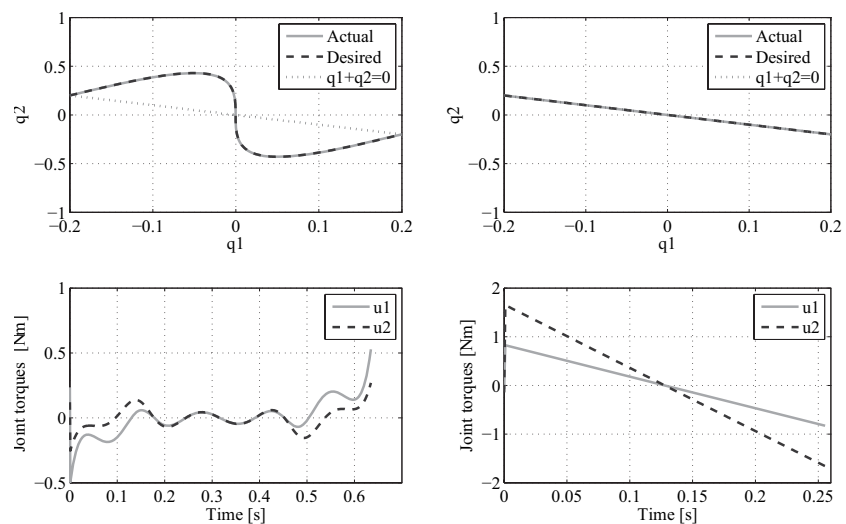

Fig. 5. Two simulation results of the velocity field tracking. The initial conditions are the same as Fig. 4, left. (Left) Tracking to the velocity field of the invariant manifold. (Right) Tracking to the "test" velocity field.

$$
\dot{q}=\alpha v+\nu
$$

and $\langle v(q), \nu\rangle=0$.

The final control input is given by

$$
u=u_{n}+u_{a}
$$

By this controller, $\nu \rightarrow 0 \quad(t \rightarrow \infty)$ is met for any initial state $\left(q_{0}, \dot{q}_{0}\right)$, which means that the velocity of the solution asymptotically along with the desired tangent vector of the manifold. (18) and (20) correspond to feedforward and feedback term, respectively. The former suffers from the modeling error, which results in poor performance of the energy preservation, while the latter does not because of the nature of feedback. See Duindam (2004) for the details. Note that, unlike in Duindam (2004), we do not need to compensate the potential term because the symmetric orbits are the solutions of the uncontrolled Hamiltonian system.

The left figures in Fig. 5 show the simulation results of this controller with the approximated tangent velocity field. The right figures show the results with the target velocity $v=[1,-1]^{T}$, which corresponds to the conservative symmetric orbits that meet $q_{1}+q_{2}=0$. The control inputs for the latter case are larger than the former. However, we can see the residual errors due to the approximation. Note that the ankle torque is used, and the energy is preserved (not shown, but see Fig. 8). If we choose a straight gait rather than the curved gait, the approximation becomes better. We expect that the error will become smaller if we increase the order of the expansion.

We refer the reader to Yamakita (2001), where a similar walking gait generation is presented, but using another velocity field control proposed by Li (1999). Different from the authors method, they attempted to follow the velocity field of the "asymptotically stable" passive walking gait (McGeer (1990)), which were approximated by an RBF network.

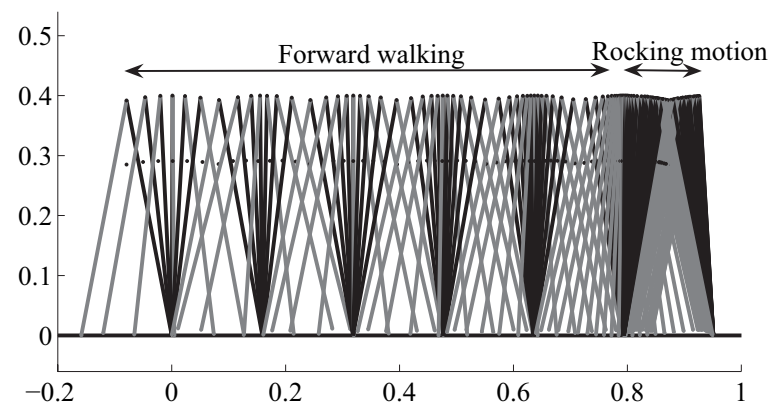

Fig. 6. Stick animation of an under-actuated symmetric walking on a level ground. The corresponding date is shown in Fig. 7.
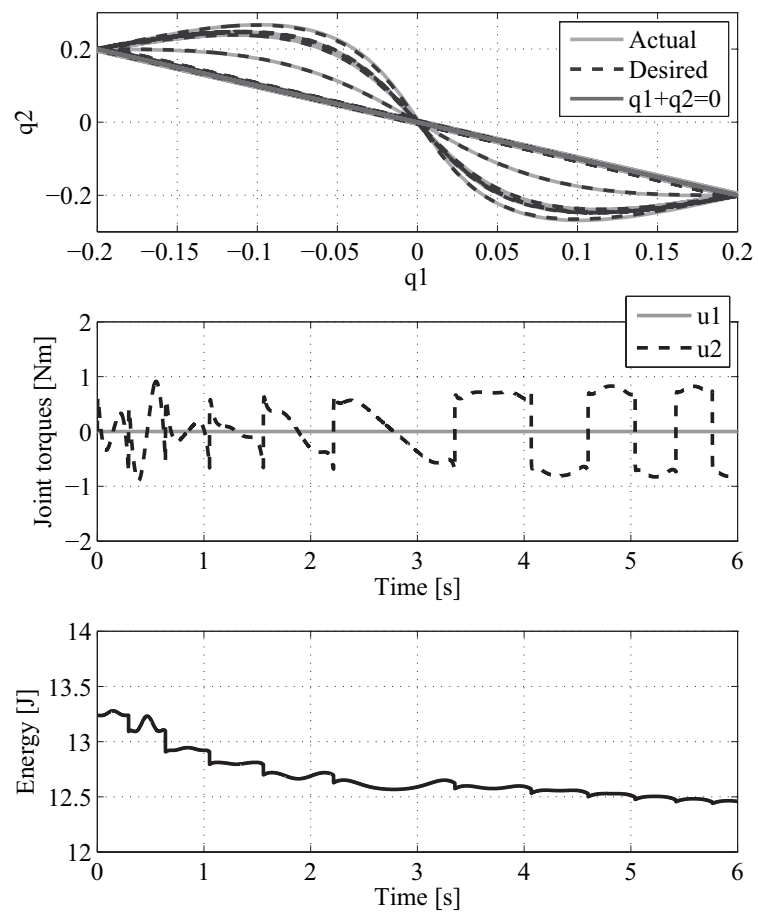

Fig. 7. Simulation result of an under-actuated symmetric walking on a level ground. The stride parameter is fixed to $\pm 0.2 \mathrm{rad}$. The robot looses the kinetic energy at every touchdown event and exhibits a few steps of rocking motions before it finally stops. The walking gaits are automatically chosen by the energy level, which are different by every step. See Fig. 6 for the animation.

\section{ASYMPTOTICALLY STABLE WALKING BY PASSIVITY-BASED CONTROL}

So far, we considered fictious lossless model to derive the invariance controller in $\mathbf{S 1}$ in our control strategy (Section 1). However, (5) does not match to the actual leg-interchanging map because there is a state transition according to the impulse equation.

Nevertheless, the invariant manifold is still powerful because we can select the trajectories of a preferred stride 

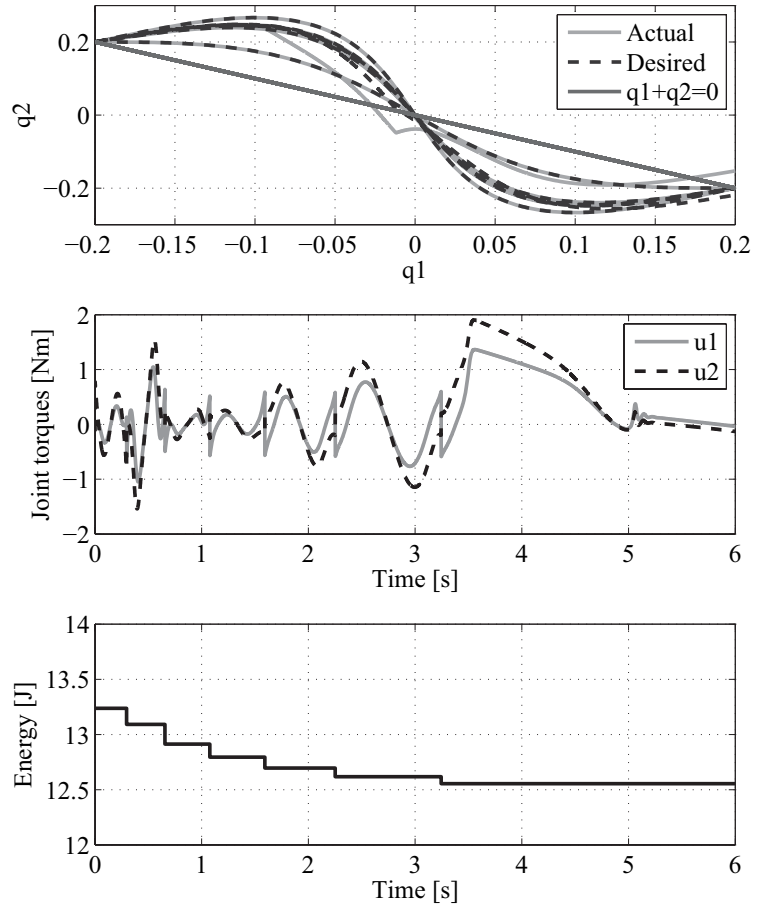

Fig. 8. Simulation result of a full-actuated symmetric walking on a level ground, in which the velocity is constrained to the tangential velocity field of the invariant manifold while the total energy is kept constant. Due to the approximation error of the invariant manifold, the control performance deteriorates at the low-speed walking.

and walking speed (energy). We can make one trajectory transit to another simply by pumping energy. The stability is ensured as long as we constrain the solutions onto the manifold. To illustrete this point, we conducted a simulation where the symmetric orbit is automatically selected according to the available internal energy, which decreases whenever the touchdown event occurs. Fig. 6 shows the result where the simple controller in Section 5.1 is used. The simulation data is shown in Fig. 7. The robot looses its energy when touchdown, then enters into the rocking motion, and finally stops at double support contact with zero kinetic energy, which is similar to our previous result by Hyon (2005). In this simulation, the norm of the input at $6 \mathrm{sec}$, was 43.844 .

On the other hand, Fig. 8 shows the simulation results of the controller described in Section 5.2. Since the approximation is not good, the robot fell within a few steps. We didn't detailed analysis to see how the performance could be improved as the approximation order increases.

We give some comments on the rocking motion. The symmetric orbit in this paper is the orbit that passes origin of the configuration space. Therefore, there is a minimum energy such that the robot can pass the potential barrier to continue walk. Otherwise, we have to consider two-periodic solution. From $(3)\left(R \circ \phi_{t}\right) \circ\left(R \circ \phi_{t}\right)=\mathrm{id}$. We find a flow $F_{r}$ such that $\left(R \circ F_{r}\right)=-$ id , and the symmetric orbits
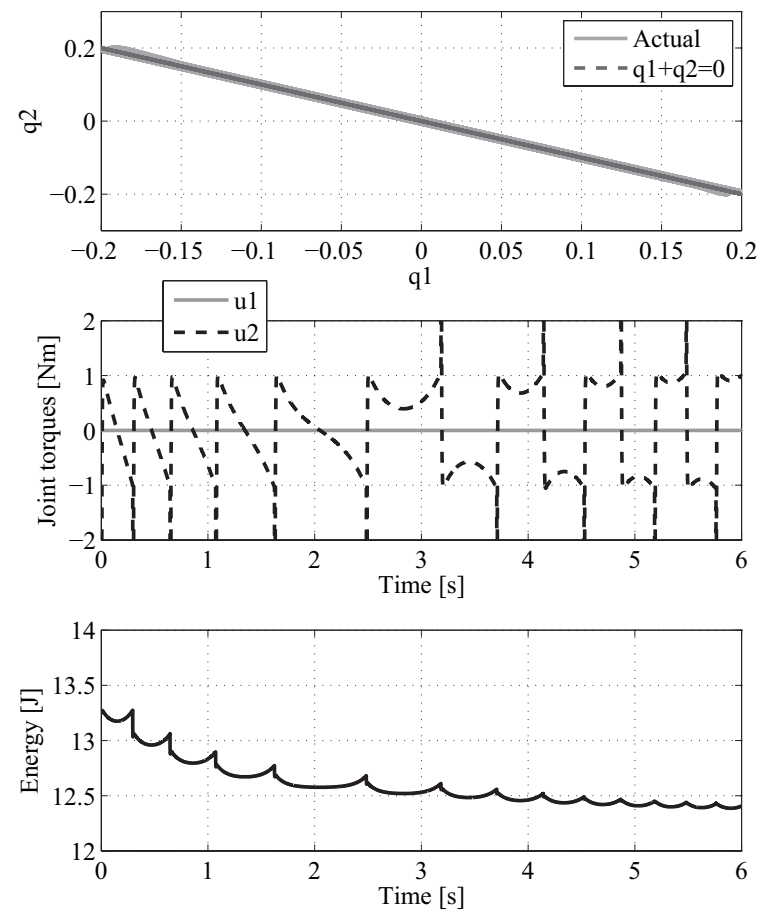

Fig. 9. Simulation result of a simple under-actuated symmetric walking on a level ground, in which the velocity is constrained to $\dot{q}_{1}+\dot{q}_{2}=0$. The walking gaits are not changing, but the speed is changing.

passing the fixed point set $\operatorname{Fix}\left(R \circ F_{r} \circ R \circ F_{r}\right)$. Then we constrain the solution to these orbits. The hybrid limit set (Grossman (1993)) where the kinetic energy is zero, is $\left\{q_{1}=q_{r}=-q_{2}\right\} \cap\left\{q_{1}=-q_{r}=-q_{2}\right\} \cap\left\{p_{1}=p_{2}=0\right\}$ Hyon (2005).

For comparison, we also did a simulation where the solution is forced to follow a single straight symmetric orbit. The result is shown in Fig. 9 (same as in Hyon (2005)). Compared to the previous results, the qualitative behaviors are not so different, but the amount of the control torque is larger. The norm of the input at 6 sec. was 147.71. Actually, the time when rocking motion occurs is 1 sec. earlier due toe the rapid decrease in the energy. This result makes sense because the uncontrolled system does not posses such a straight symmetric orbit.

Now we are in position to derive a passivity-based controller to asymptotically stabilize the periodic orbits with desired walking speed. Similar to simple pendulum, we can stabilize periodic orbits by adding or deleting the energy. This corresponds to our motion control strategy (S2) in Section 1. As an example, let us consider stabilization by the ankle torque. As shown in Section 5.1, the stability is ensured even if the ankle torque is zero, we only have to pump the energy into the system by applying the ankle torque. As a simple controller, we can use a proportional feedback law

$$
u_{1}=-K_{E} \dot{q}_{1}(E-\bar{E})
$$




\section{ACKNOWLEDGEMENTS}
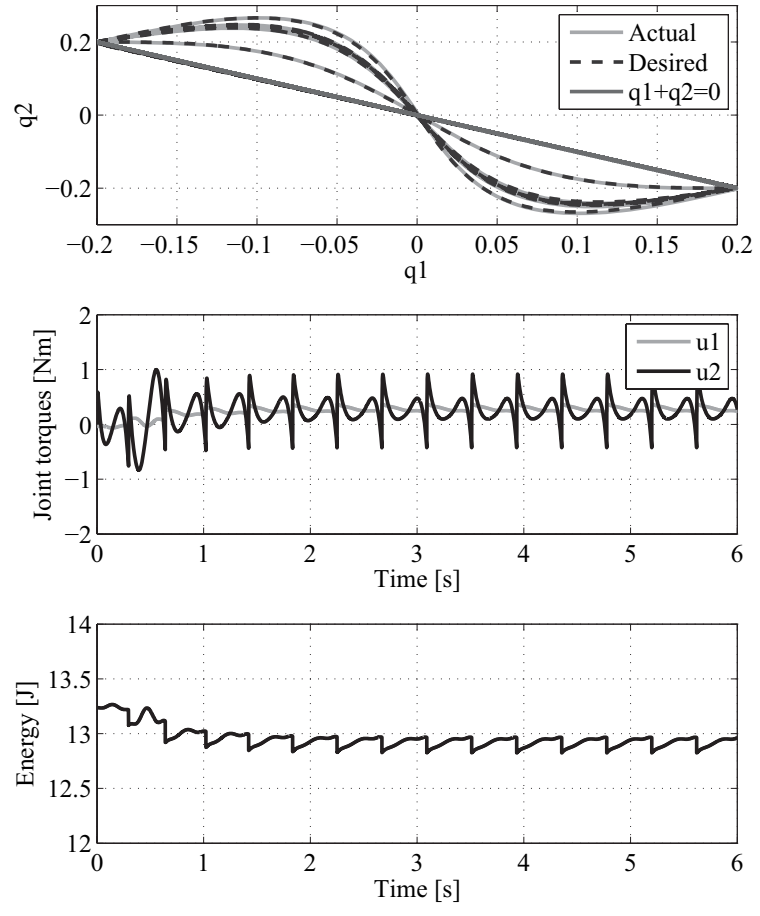

Fig. 10. Simulation result of a stable walking. A simple ankle actuation is used for controlling the energy.

for some target energy $\bar{E}$ with $K_{E}>0$. Fig. 10 shows the simulation result. It can be seen that an asymptotically stable walking has been achieved.

\section{SUMMARY}

This paper proposed novel walking gait generation for a double-pendulum-like biped walking model based on the family of the symmetric orbits. The effectiveness of the proposed method was confirmed through the numerical simulations.

By introducing an involution $R$ associated with the leg switching map, we allowed the flow of the saddle-center dynamics of the uncontrolled pendulum to possess the timereversal symmetry with respect to $R$, and the conjunction of the flow and $R$ to form a family of symmetric orbits parameterized by the orbital energy and the stride. The invariant manifold of the family of symmetric orbits was obtained approximately using a perturbation method. By constraining the solution onto the invariant manifold using the control inputs, stable walking gaits were generated in a semi-global manner. Based on the passivity of the closed-loop system, a robust speed-controlled walking was achieved in a very simple way.

Our current work includes the detailed analysis on the error propagation, learning optimal walkin control by using iterative learning Satoh (2008), as well as the scaling up the current method to realistic biped model shown in Hyon (2005).
The authors gratefully acknowledge the contribution of Prof. Kazuyuki Yagasaki (Gifu University, Japan) in computation of the invariant manifold.

\section{REFERENCES}

Devaney, R.L. (1976). Reversible diffeomorphisms and flows. Transactions of the American Mathematical Society, 218, 89-113.

Duindam, V., Stramigioli, S., and Scherpen, J. (2004). Passive compensation of nonlinear robot dynamics -. IEEE Transactions on Robotics and Automation, 20(3), 480-488.

Grizzle, J., W., Abba, G., and Plestan, F. (2001). Asymptotically stable walking for biped robots: Analysis via systems with impulse effects. IEEE Transactions on Automatic Control, 46(1), 51-64.

Grossman, R, L., Nerode, A., Ravn, A, P., and Rischel, H.a. (1993). Hybrid systems. Lecture Notes in Computer Science 736. Springer.

Guckenheimer, J. and Holmes, P. (1983). Nonlinear Oscillations, Dynamical Systems, and Bifurcations of Vector Fields. Springer.

Hyon, S., Emura, T., and Ueta, T. (2004). Detection and stabilization of hybrid periodic orbits of passive running robots. In Mechatronics and Robotics, 13091314. Aachen, Germany.

Kousaka, T., Ueta, T. and Kawakami, H. (1999). Bifurcation of switched nonlinear dynamical systems. IEEE Trans. Circuits and Systems, CAS-II(7), 878-885.

Hyon, S. and Emura, T. (2005). Symmetric walking control: Invariance and global stability. In IEEE ICRA, 1455-1462.

Lamb, Jeroen, S.W. and Roberts, J.A.G. (1998). Timereversal symmetry in dynamical systems: A survey. Physica D: Nonlinear Phenomena, 112(1-2), 1-39.

Li, P. and Horowitz, R. (1999). Passive velocity field control of mechanical manipulators. IEEE Transactions on Robotics and Automation, 15(4), 751 - 763.

Marsden, Jerrold, E. and Ratiu, Tudor, S. (1999). Introduction to Mechanics and Symmetry: A Basic Exposition of Classical Mechanical Systems. Springer-Verlag.

McGeer, T. (1990). Passive dynamic walking. The International Journal of Robotics Research, 9(2), 62-82.

Morita, S., Fujii, H., Kobiki, T., Minami, S., and Ohtsuka, T. (2004). Gait generation method for a compass type walking machine using dynamical symmetry. IEEE/RSJ IROS, 2825-2830.

Sevryuk, M.B. (1986). Reversible Systems. Lecture Notes in Mathematics 1211. Springer.

Wieber, P.B. and Chevallereau, C. (2006). Online adaptation of reference trajectories for the control of walking systems. Robotics and Autonomous Systems, 54(7), 559566.

Yamakita, M. and Asano, F. (2001). Extended passive velocity field control with variable verocity fields for a kneed biped. Advanced Robotics, 15(2), 139-168.

Satoh, S., Fujimoto, K. and Hyon, S. (2008) A framework for optimal gait generation via learning optimal control using virtual constraint. IEEE/RSJ IROS, 3426-3432. 